\title{
Using a network of the whole population of the Netherlands to measure exposure to differing educational backgrounds
}

\author{
Jan van der Laan* Marjolijn Das*† Saskia te Riele* \\ Edwin de Jonge* Tom Emery ${ }^{\dagger}$
}

May 13, 2021

\begin{abstract}
In this analysis we present a whole population network which uses administrative data to construct a network incorporating 1.4 billion relationships between the 17 million inhabitants of the Netherlands. Relationships are identified between individuals who live in the same household, live close to each other, work for the same company, attend the same educational institution, or belong to the same extended family. This network has properties that are rare in observed social networks, which opens up new applications for network science in the social sciences. To demonstrate the applications of such a network, we use a random walk approach to estimate exposure to individuals of differing educational backgrounds and whether specific types of relationships increase or decrease this segregation. The results suggest that household relationships greatly increase segregation whilst work, school and neighborhood networks relationships increase exposure to individuals with different backgrounds. The size of these effects is context dependent. Further applications of a whole population network are also discussed.
\end{abstract}

*Statistics Netherlands

${ }^{\dagger}$ Erasmus University Rotterdam 
Keywords: Networks, Exposure, Random Walk, Education, Segregation

\section{Main}

Relationships are a vital element of people's lives and link them to their partners, children, friends, colleagues, and neighbors. This complex web of relationships exposes us to different types of people, whilst segregating us from others. Computational social science can help to better understand this complexity and network analysis has become a pervasive methodology for understanding complex social systems and group dynamics $[1,2,3,4,5]$.

Existing social network research is limited by a lack of extensive and detailed population data in most countries. This limits the external validity of much social network analysis and hinders the integration of network science into substantive research of social phenomena. In countries where governments do collect such data, the highly sensitive nature of this information places ethical and legal restrictions on its usage. In the Netherlands, the national data infrastructure for Social Science (ODISSEI; www.odissei-data.nl) has constructed a highly secure environment which allows for the analysis of government population data via the national supercomputer that is capable of large scale network analysis.

Using this secure environment we have created a whole population network. The network is derived from governmental administrative registers held by Statistics Netherlands that can be linked at the individual level [6]. Data sets include the population register, with information on family relationships and geographical location of the addresses, tax registers with information on jobs, wages and employers and registers with information on 
enrollment in and diplomas from educational institutes ${ }^{1}$.

In accordance with the law which governs Statistics Netherlands conduct ${ }^{2}$ and the General Data Protection Regulation ${ }^{3}$, the data are protected by extensive rules and regulations with respect to privacy to ensure that no information about individual persons is disclosed in any output from these data sets, and individuals can never be recognized in the output. This applies to all analyses presented here ${ }^{4}$.

The nodes of our network are all persons in the official Dutch population register on October 1st 2018. The links are family relations, household membership, neighbors, colleagues and class-mates, also as of October 1st 2018. Since it cannot be inferred whether people actually interact with others in their network, the network is a reservoir of potential contacts and exposure rather than a realized network. Additionally, friends and other contacts via church, sports or hobby clubs are not included, and neither is subjective information such as motivations, attitudes and quality of relationships ${ }^{5}$.

Family layer The population register contains the legal (i.e. biological or adoptive) parents of all persons whose parents were also registered in the

\footnotetext{
${ }^{1}$ The data on educational diplomas are available for $68 \%$ of the whole population and $74 \%$ of the research population aged $25-55$. The probability of a missing value increases with age and is higher for non-Dutch born. All the other data are available for the complete Dutch population of roughly 17 million inhabitants.

${ }^{2}$ https://wetten.overheid.nl/BWBR0015926/2018-07-01

${ }^{3}$ Regulation (EU) 2016/679 (General Data Protection Regulation)

${ }^{4}$ Full and detailed information about the security measures and data privacy protections in place for the analysis of microdata for research can be found here: https://www.cbs.nl/en-gb/our-services/customised-services-microdata/

${ }^{5}$ It should be noted that CBS and ODISSEI do collect such data via surveys and linkage keys exist to integrate this data into the analysis for specific sample populations.
} 
Netherlands in 1995 or later. The link between parents and children is therefore most complete for persons born since 1966. We derived the following family relations: 'child', 'parent', 'grandparent' (parent of a parent), 'grandchild' (child of a child), 'sibling' (child of a parent), 'co-parent' (parent of a child), 'niece/nephew' (child of a sibling), 'aunt/uncle' (sibling of a parent) and 'cousin' (child of an aunt/uncle). In the case of 'sibling', 'full-siblings' (siblings that share two parents), 'half-siblings' (siblings that share only one parent), and 'siblings (unknown)' (siblings that share one parent, but for at least one of the siblings one of the parents is not known) are all included in the analysis. Note that family relations by marriage, such as the husband of an aunt, a step sibling or parents-in-law, were not included in the family layer.

Household layer The household database contains information on the household to which each person belongs. Statistics Netherlands builds this database by combining the address information from the population register with information on direct family relations (parents, marriages), tax data (shared income tax filings), and survey data. The household database contains the type of household (e.g. 'married couple with children') and the position of the persons in the household (e.g. 'partner', 'child'). We used the household database to derive relationships between household members: 'partner', 'household member' and 'institutional household member' (for members of institutional households, e.g. care homes, which can be very large).

Neighbors layer For each household member, 'neighbors' were defined as the members of the geographically closest ten households within fifty meters. 
When more than ten households meet these conditions, households were randomly chosen in order of increasing distance. Institutional households with four persons or less were treated as above. In institutional households with more than four persons, each person was considered a separate household when determining neighbors.

Colleague network For all employees we first identified the company of employment from income tax data (Polisadministratie) and then derived all colleagues of each person. There are large differences in the sizes of companies which range from single person businesses to large corporations and institutions such as government agencies. It was decided to limit the colleague layer to the one hundred colleagues who are geographically closest in terms of residence for two reasons. First, it is unrealistic that a person actually meets all colleagues in large companies. Second, many of the large companies have different locations (for example retail chains, supermarkets, banks etc). The register data do not include information on work location. By selecting colleagues who live close by, we increase the probability that they work at the same location and come into contact with each other. However, this remains an approximation.

Class-mates network For persons enrolled in primary, secondary, vocational and higher education on 1st January 2018 it was determined which school and location they attend, which type of education they are following and in what grade they are. This information was used to create networks of grade peers, the closest possible approximation of class. Multiple classes with children in the same grade in the same location, common in primary education and the lower years of secondary education, cannot be distinguished 
in the data. The later years of secondary education and higher education are organized around individual study programs, meaning that students likely mix across the entire year group.

To demonstrate the value of a whole population network analysis, we examined the degree of segregation by educational attainment within the dutch population. Educational outcomes are shaped and heavily influenced by resources such as parental income, but also social and cultural capital [7, 8]. For this reason, there are concerns about increasing inequalities between the lower and the higher educated and of a growing segregation brought about by deepening divides between those in society who have high levels of education and those who do not [9]. Growing educational segregation may lead to polarization, fewer opportunities for social mobility, and decreasing social cohesion [10]. In this paper we illustrate the value of a whole population network by analyzing segregation by education level.

Not all forms of segregation and network limiting are necessarily unfavorable as small and homogeneous networks can be efficient [11, 12], however, there is consensus among most social scientists and policy makers that extensive and widespread segregation within a society is undesirable. 'Weak ties' or 'bridges' are thought to be especially important for social mobility and cohesion: relationships with people that belong to a different social group $[13,14]$ or with people that are one step removed from one's social circle, such as friends of friends $[15,16]$.

Educational segregation has been extensively studied before, especially the geographical dimension of residential segregation $[17,18]$. The highly educated, for example, are over represented in and around large cities due to selective migration for education opportunities and specialized knowledge 
work $[19,20]$. However, such analyses only focus on one dimension of segregation or what we refer to in network terms as a layer.

The higher and lower educated also move in different social circles at work and in their free time [21, 22], are less likely to marry each other [23] and send their children to different schools. This warrants the broader view of educational segregation encompassing multiple dimensions and layers within society, as argued elsewhere $[24,25,26]$.

Given these findings, a whole population network can help us better understand what types of relationships and links strengthen and dilute the segregation of educational groups. In this paper we showcase the possibilities by analyzing the segregation of different educational groups in strongly urbanized versus lesser urbanized and rural municipalities in the Netherlands. This provides insight into the relationship between residential segregation and other types of segregation (in other network layers) and the role of urbanicity as a main driver for selective migration, as well as insight in segregation within cities beyond the well known residential segregation.

\section{Methods}

\subsection{Descriptive Statistics of the network}

The total network contains 17,254,523 nodes. There are more than 1.4 billion links, a summary of which can be seen in table 1 . The mean degree for each node across the whole network is 82.7 with a median of 61 .

Based on its construction and these parameters, we argue that this whole population network is distinct from most networks used in social network science. It includes a complete list of individuals (nodes) and potential rela- 
Table 1: Summary statistics of degree distribution for each relationship type

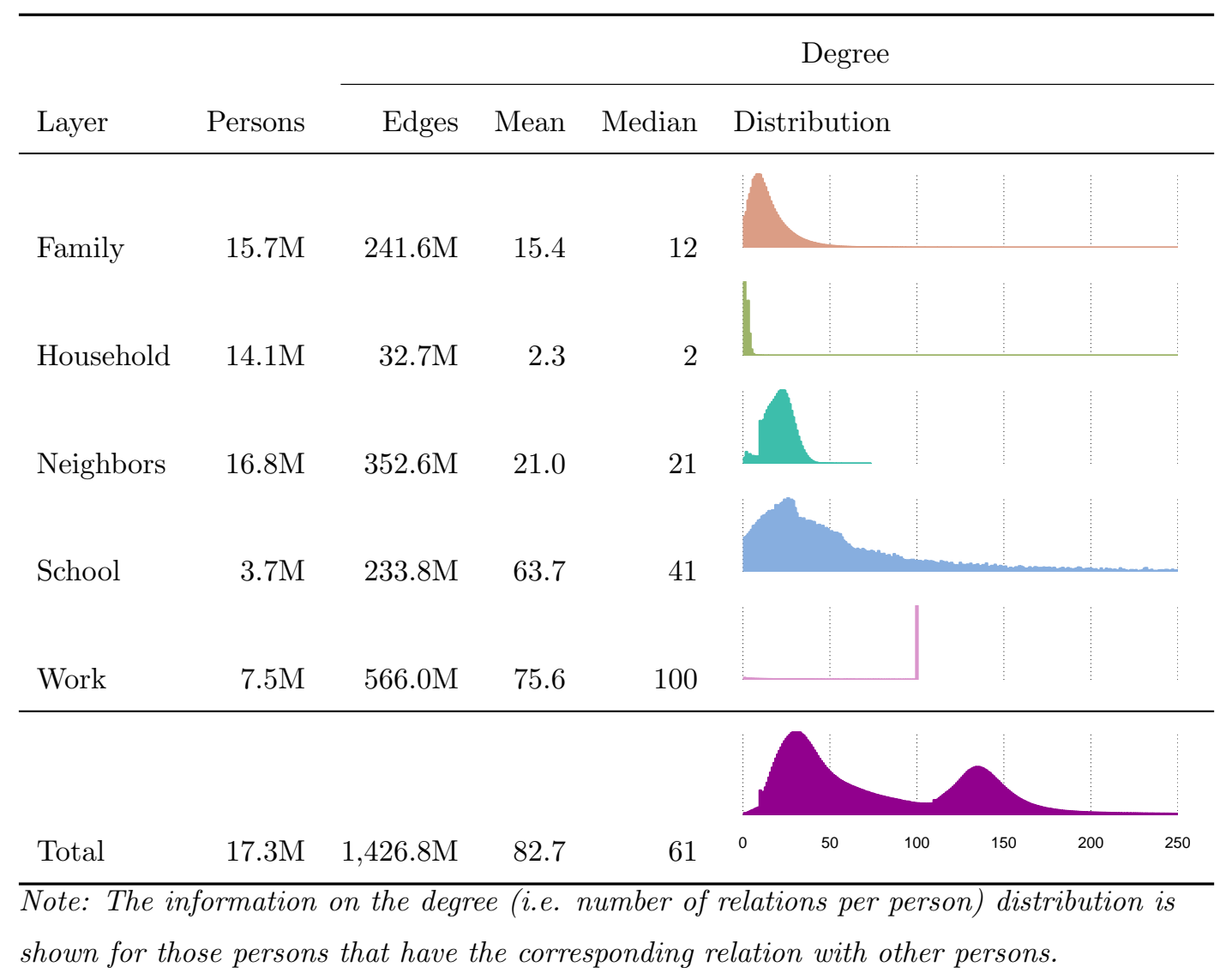


tionships (links) within a geospatial area (The Netherlands). This is distinct from traditional social network analysis which generally relies on a limited number of relationships existing within a single network or sector (i.e. a company, social media platform or community). This whole population network combines several network layers covering distinct social institutions into a multiplex network.

A large proportion of nodes have links in all layers of the network and the number of degrees in each layer is not correlated (see table 2), suggesting that there is a high degree of independence between layers. The multiplex nature of the whole population network raises the potential for studying inter-dependency between network layers [27].

What is also notable is that the inclusion of individuals in a whole population network is not conditional on the existence of relationships within any given network layer. A whole population network includes all individuals within a population, irrespective of their participation in specific layers, so unconnected nodes are included too. The result is a diffuse network without nodes with high centrality [28].

The complete network thus represents the Dutch population and all relationships within these layers: the structural connections within a society based on family, work, school and living environment. This results in greater

external validity for subsequent analysis. It is a unique tool for understanding the relevance of network dynamics for society as a whole.

\subsection{Used classifications}

In presenting the results, a number of classifications were used. First, the education levels used in the analysis are in line with International Standard 
Table 2: Degree-degree correlations for each relationship type

\begin{tabular}{lrrrrr}
\hline Layer & Family & Household & Neighbors & School & Work \\
\hline Family & 1.000 & 0.053 & 0.022 & -0.018 & 0.118 \\
Household & 0.053 & 1.000 & 0.102 & 0.097 & -0.009 \\
Neighbors & 0.023 & 0.103 & 1.000 & 0.046 & 0.081 \\
School & -0.019 & 0.097 & 0.046 & 1.000 & -0.052 \\
Work & 0.118 & -0.009 & 0.081 & -0.052 & 1.000 \\
\hline
\end{tabular}

Classification of Education [29] (ISCED 2011) such that Lower Education corresponds to ISCED $0,1, \& 2$; Intermediate education corresponds to ISCED $3 \& 4$; Bachelor or equivalent corresponds to ISCED $5 \& 6$ and Masters or higher corresponds largely to ISCED $7 \& 8$ with the exception of a very small group who belongs in ISCED 6. Second, some of the results are geographically normalized at NUTS-3 level. This refers to the European Unions Nomenclature for Territorial Units [30]. NUTS-3 is approximately equivalent to county level in the United States. Third and finally, some of the results are presented against the degree of urbanization. This is based on the average surrounding address density in a municipality. The average surrounding address density is the average number of addresses within a one kilometer radius around an address. The degree of urbanization is classified in five categories: Very strongly urbanized: 2,500 addresses or more per square kilometer; Strongly urbanized: 1,500 to 2,000 addresses per square kilometer; Moderately urbanized: 1,000 to 1,500 addresses per square kilometer; Hardly urbanized: 500 to 1,000 addresses per square kilometer; Not urbanized: fewer than 500 addresses per square kilometer. 


\section{$2.3 \quad$ Segregation scores}

We measured segregation using the exposure and isolation index proposed by Massey \& Denton [31] and the random walk-based segregation measure proposed by Ballester \& Vorsatz [32]. This method has several advantages. First and most importantly, a network is an integrally connected entity and each relationship should not be looked at in isolation. 'Weak ties' or 'indirect ties' and more generally pathways from one person to others, are key for the dissemination of knowledge, culture, behavior etc through a network.

Second, it provides an opportunity to measure educational segregation at both the individual level and a group level of choice. As Peel et al. argued [33], segregation can vary strongly between individuals and over the network. Segregation should, therefore, be measured at the individual level and can then be further aggregated when necessary. It also opens up the possibility of studying the relationship between segregation and other individual level outcome variables. Third, compared to the more simple method of measuring only direct contacts of a person, the random walk approach gives a more precise score when the number of direct contacts is small.

As is standard in segregation research, the scores were normalized to account for differences in group size. We used two normalization schemes, that are both informative: a national segregation score normalized to the country level and a regional normalization at sub-regional levels (NUTS-3) to address the regional differences in demographics. The segregation analyses were restricted to persons aged between 25 and 55 in order to minimize missing values for education level. Note however that we used the links of the total network to calculate segregation, that is, connections via one or more persons who are outside this age range were also taken into account. 
Our method consists of five steps, outlined below. First, the weights of the outgoing links per person were calibrated so that they sum up to one and each network layer gets an equal total weight. Second, for each individual $v$ we derived an ego-network where individuals closer to $v$ and with more pathways to $v$ received a higher weight. Third, for each individual $v$ we calculated its exposure $e_{v}$ to other groups in its ego-network. Fourth, $e_{v}$ was normalized for differences in group sizes and transformed to obtain the individual segregation score $s_{v}$. Fifth, we aggregated $s_{v}$ to obtain median segregation scores for the different subgroups and compared those.

Step 1. When there is a relation between the individuals $v$ and $u$, a positive weight $A_{v u}$ exists that reflects the strength of the relation between $v$ and $u$. As was mentioned in section 2, the network consists of five layers. A person $v$ is present in $n_{v}$ layers. For example, when a person has family, household members and neighbors but does not go to school or have a job, $n_{v}=3$. Since the relative importance of different layers is yet unknown, we parsimoniously attributed the same weight to all connections within the same layer and let the sum of the weights within a layer add up to $1 / n_{v}$. Therefore, the total sum of the weights for one person equals one $\left(\sum_{u} A_{v u}=1\right)$ which is necessary for the random walk (next step) and each layer was deemed equally important for each person.

Step 2. Starting from an individual $v$ one starts to follow random connections. A connection from individual $v$ to individual $u$ is followed with a probability $A_{v u}$. After every step there is a probability $1-\alpha$ of stopping and a probability $\alpha$ of continuing the random walk. The probability of stopping at person $u$ when starting the random walk at $v$ is given by $P_{v u}$. Methods of calculating $P_{v u}$ given $\alpha$ are given by Ballester et al. [32] and Van der Laan 
et al. [34].

For each individual $v$ this leads to an ego-network where individuals $u$ closer to $v$ (in terms of number of steps between $v$ and $u$ ) are included in the segregation score with a larger probability than individuals that are further away. This is a desired property, since it is likely that individuals that are close (in network terms) exert a larger influence on $v$ than individuals that are several steps removed from $v$. For small values of $\alpha, v$ is predominantly exposed to only the direct neighbors. For large values of $\alpha, v$ will be exposed to a large portion of the network. Following Ballester et al., we used a fixed value of $\alpha=0.85$.

Step 3. These weights $P_{v u}$ can then be used to calculate a weighted exposure of $v$ to other groups. However, in the case of the education level, for approximately $26 \%$ of the population under study information on education is missing. To correct for these missing values, the data set contains weights, $w_{v}$. Although these weights are determined for the simple goal of determining population totals, Van der Laan et al. [35] demonstrated that these weights can be incorporated as an additional term in the equation. The weights were set to zero when the education level is not observed. Therefore, the individual exposure of $v$ to persons with other education levels than $v$ is given by:

$$
e_{v}=\frac{\sum_{u} w_{u} P_{v u}\left[g_{v} \neq g_{u}\right]}{\sum_{u} w_{u} P_{v u}},
$$

when the education level of $v$ is observed and missing otherwise. Here $g_{v}$ is the group of $v$ and $[x]$ is 1 when $x$ is true and 0 otherwise (Iverson bracket notation). As can be seen, the individual exposure is the weighted fraction of persons from a group other than the group of person $v$.

The weights $w_{v}$ were also used to focus our analyses to our target pop- 
ulation of persons aged between 25 and 55 by giving persons younger than 25 and older than 55 a weight of zero. In that way they still contributed to the network during the random walk (e.g. they contributed to $P_{v u}$ ) but not when calculating the individual exposure.

Step 4. Even when there is no segregation, e.g. when the connections between persons are purely random, we would expect that the individual exposure of $\mathrm{v}$ is $1-f_{g_{v}}$ with $f_{g_{v}}$ the relative size of the group of $v$ in the population. For example, the individual exposure to "others" of highly educated persons in a population with 10\% highly educated persons is expected to be $90 \%$ in case of random connections. In order to be able to compare the exposure across groups, we should correct for the size of the groups. We normalized both on the country level and on the NUTS-3 level. We subsequently subtracted the normalized individual exposure from 1 to get the inverse of exposure: segregation. This leads to the individual segregation score for $v$ :

$$
s_{v}=1-\frac{e_{v}}{1-f_{g_{v}}} .
$$

This score equals 1 for an individual that is not exposed to other groups (complete segregation), equals 0 when the exposure is as expected based on the group sizes (no segregation) and can be smaller than 0 when an individual is exposed more to other groups than expected. This score is strongly related to the $\eta^{2}$ score used to measure segregation with aggregated data (see e.g. Massey and Denton [31]).

Step 5. The weighted median was calculated from the individual segregation scores, using $w_{v}$ as weights. 


\subsection{Layer importance}

It is not only of interest to measure the individual segregation, but also to measure how each layer contributes to the individual segregation scores. This was done using Shapley values. These scores originate from game theory, where they measure the contribution of the members in a team to the overall success of the team [36]. The Shapley values are also used in machine learning to measure the importance of predictors for individual predictions [37]. To measure the effect of a certain layer, networks were built using all combinations of layers excluding the layer of interest and then, normalized segregation scores were calculated. After that, the layer under study was added to each of the possible networks and the change in the segregation scores was measured. A weighted mean of these changes over the different networks gives the total effect of the layer on the individual normalized segregation scores.

It is here that the distinct value of a whole population network is also evident. The removal of a specific layer not only removes the immediate links that an individual has within a layer but also removes it from their wider network. For example, when we remove the neighborhood layer to calculate the Shapley value, we not only remove the links an individual has to their neighbors but also all the pathways that link the individual to others indirectly via neighbors. If an ego is exposed to higher educated people because ego's colleague lives in an area with highly educated people, this indirect exposure will automatically be removed when the neighborhood layer is removed. Hence, the analysis is broader than the individuals' personal networks: it focuses on the pervasive structure of the layers.

One of the advantages of using Shapley values is that the effects of the 
individual layers add up to the total segregation score:

$$
s_{v}=s_{v}^{(\text {family })}+s_{v}^{\text {(household) }}+s_{v}^{\text {(neighbors) }}+s_{v}^{(\text {work })}+s_{v}^{\text {(school) }}
$$

The Shapley values do not reflect the segregation score of the layer in question but reflect whether this layer has an increasing or decreasing effect on the total segregation score.

\subsection{Software}

Initial calculations were performed using the matrix libraries [38] available in $\mathrm{R}$ [39] on the ODISSEI secure super computer [40]. Later computations were performed using custom software written in $\mathrm{C}++$ on servers at Statistics Netherlands. Using the custom written software a single computation of the exposure takes approximately two hours and less than 100GB of memory. The custom software code is available on request.

\section{Results}

\subsection{Educational Segregation}

Segregation scores were calculated for all 6,762,163 individuals aged 25-55 using the random walk approach. This estimated the exposure to individuals of different educational levels to ones own within ones own personal network. Segregation was taken as the inverse of exposure. This score equals 1 for an individual that is not exposed to other groups (complete segregation), equals 0 when the exposure is as expected based on the group sizes (no segregation) and can be smaller than 0 when an individual is exposed more to other groups than expected. 


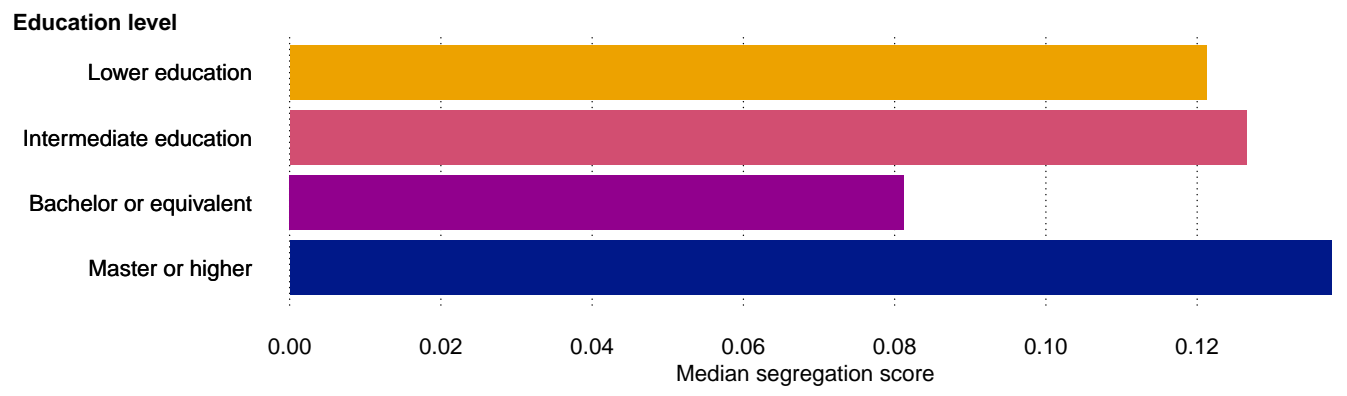

Figure 1: Median normalized exposure scores for the difference education levels.

We then examined the degree to which this segregation score differed over individuals of different educational levels and the urbanicity of the local area. Subsequently we assessed how the score changed when various layers of the network were introduced or excluded using Shapley values. This allows us to identify which layers increase segregation and which reduce it. The analysis in this use case are demonstrative with the results showing sensitivity to decisions regarding the weighting of specific layers and the definitions applied to specific relationship types such as colleagues and neighbors. Any substantive inferences derived from these analyses should be subject to extensive sensitivity analysis.

\subsection{Segregation scores}

Figure 1 shows the median segregation scores as a function of education level on the national level. People with a master's degree or higher were the most segregated from individuals of other educational levels, while people with a bachelor's degree were the least segregated.

Figure 2 shows segregation scores by education and the degree of urban- 


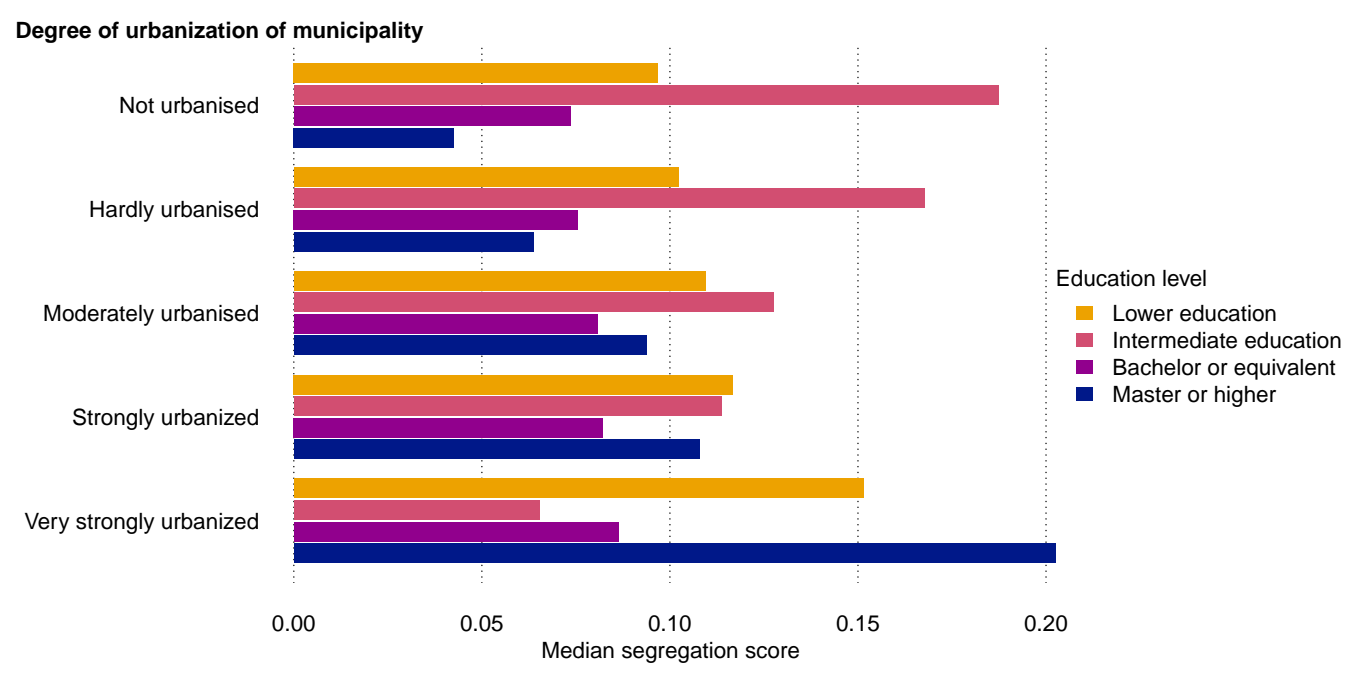

Figure 2: Median normalized exposure scores for the difference education levels as a function of degree of urbanization.

ization (see 2.2) of the municipality. Those with a master's degree were most segregated in the most urbanized municipalities and this decreased with decreasing urbanization. The lower educated also had the highest segregation scores in highly urbanized municipalities. Segregation of those with an intermediate education on the other hand increased with decreasing urbanization.

People with a university education (i.e. master's degree or higher) predominantly reside in urban and suburban areas. Since all layers of the network have geographical constraints, it is highly likely that the share of university educated in the networks of urban residents will be larger than the country's average. This would automatically lead to higher segregation levels of the university educated in cities. Geographical distribution of lower and intermediate educated may similarly color the results. Normalization at the NUTS-3 level (see 2.2) provides insights in segregation patterns above and 


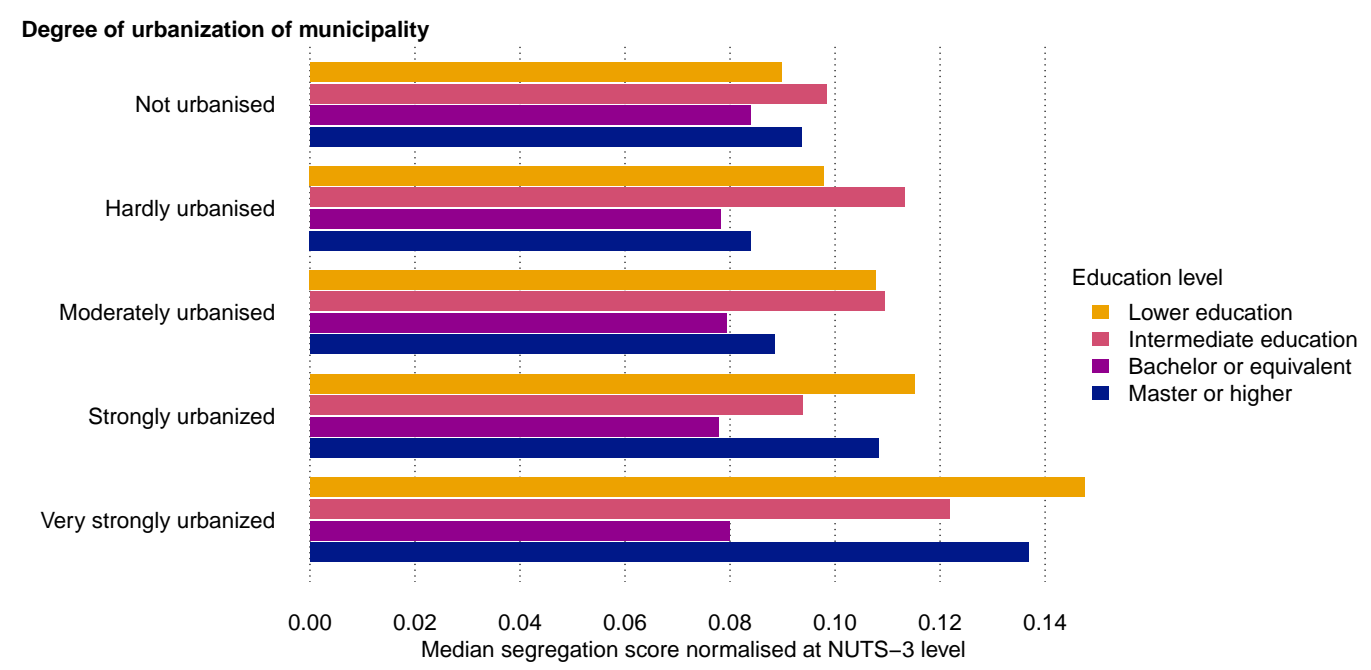

Figure 3: Median normalized segregation scores for the different education levels as a function of degree of urbanization. Normalized to NUTS-3.

beyond geographical segregation across regions.

Figure 3 shows segregation scores by education level and degree of urbanization, normalized at the NUTS-3 level. The negative relationship between urbanization and segregation for mid-level educated has now completely disappeared. These were caused mainly by the geographic distribution of this education level. The segregation scores of those with a masters or higher have decreased but remain relatively high in highly urbanized municipalities. Segregation of the lower educated does not change much. The highest segregation scores are still in the most urbanized municipalities. Segregation of people with a bachelor's degree remain unchanged, and they are consistently less segregated than other groups. The high segregation in strongly urbanized municipalities of both lower and highly educated fits the existing literature that large Dutch cities are segregated [41, 42]. 


\subsection{Layer Contributions}

Using Shapley values, the influence of different layers can be established (Figure 4). The household layer had a strong positive effect on segregation scores that was largely independent of urbanization degree. This is not surprising since in this age group, most household members are partners (34.5\%) and children (52.5\%). Social science literature shows a strong tendency for assortative mating or homogamy with respect to education. Homogamy can be brought about by preferences, meeting opportunities in educational institutes, and/or men and women striving for a partner with resources [43, 44].

The impact of the household layer is influenced by our decision to weight each layer equally, irrespective of the size. Household layers are relatively constrained in size and therefore the weight attributed to this layer places significant emphasis on a small number of individuals. The colleague network by contrast is likely to be larger, increasing the likelihood of at least some educational diversity and yet carrying the same statistical weight as the household network. A strong feature of the household layer is that it consists of individuals with who the ego almost certainly interacts, rather than reflecting potential relationships as is the case for the other layers.

The influence of the family layer is largely in line with the social mobility literature. For the lower and midlevel educated in more urbanized municipalities, the family layer increased segregation which would suggest low social mobility as family members were likely of the same educational background as them. In less urbanized municipalities, the role of family reversed and it became a means by which individuals were exposed to people from differ-

ent backgrounds, perhaps because family members themselves had attained higher levels of education and this was a primary means of exposure to other 
groups.

For those with a master's degree or higher, the family layer lowered segregation most strongly in highly urban municipalities, suggesting high social mobility as the family layer exposed them to individuals with lower educational levels than themselves. This may be related to migration background (including rural to urban migration and international migration) as people with a migration background predominantly live in cities. The university educated among them may often be the first of their family to complete a university education, especially through a Dutch university. This is in line with the educational expansion observed in many countries over the past 30 years which has seen many first generation university students.

The overall segregation scores show that strongly urbanized municipalities are more segregated than less urbanized municipalities. In very strongly urbanized municipalities, the neighbors layer increased segregation and the work layer was almost neutral for those with a masters degree or higher. For the lower educated on the other hand, these same layers decreased segregation in very strongly urbanized municipalities. In other i.e. less urbanized municipalities neighbor and work layers decreased segregation for all groups. This is in line with the idea that cities have more residential segregation and a highly specialized knowledge economy [17].

The schools layer hardly had any influence on segregation scores, but where it did there was a small decrease in segregation suggesting that the schooling system does offer a degree of exposure to others from differing educational backgrounds. Note that in our population aged 25-55 the school network exerted its influence mainly through its indirect links (e.g. parents of the classmates of the children of the ego), not directly since hardly any 


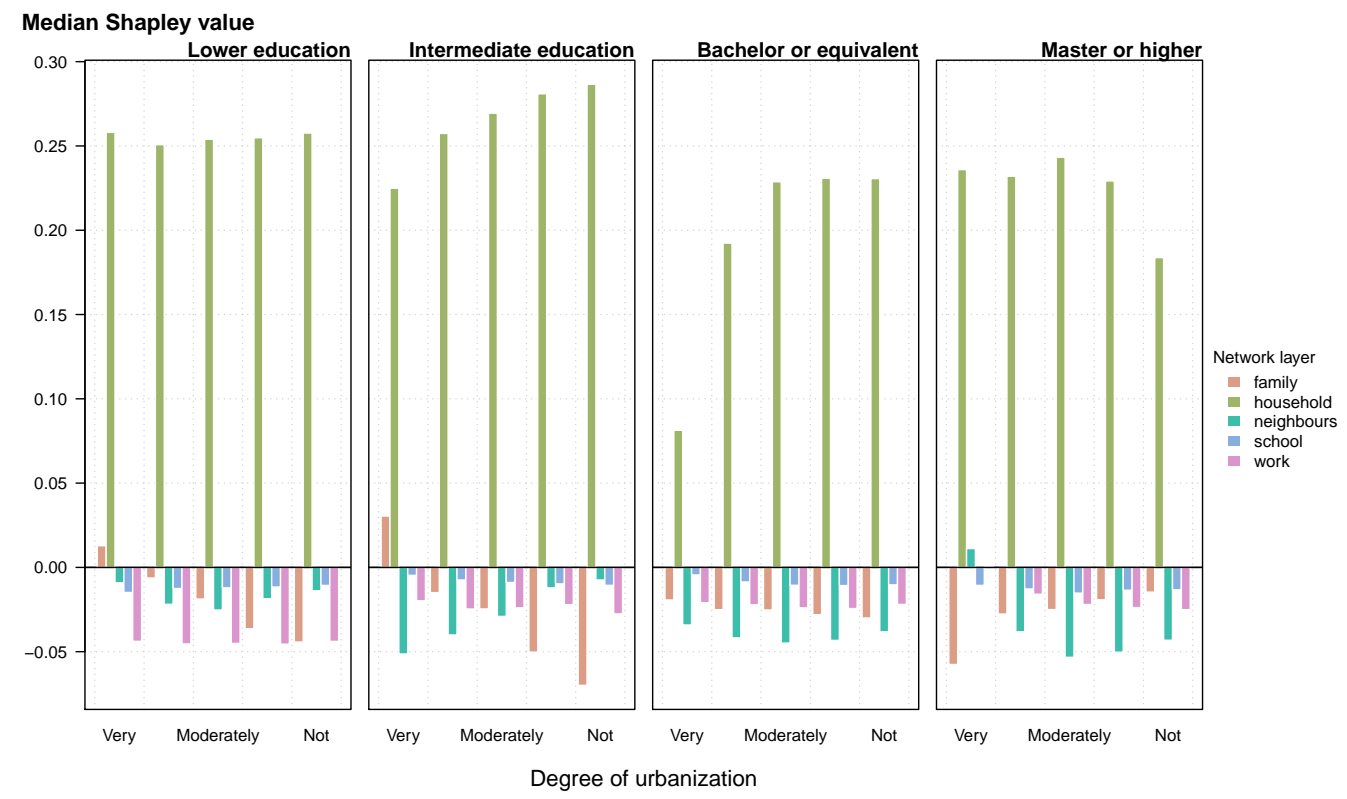

Figure 4: Median Shapley values for the different education levels as function of degree of urbanization. Normalized to NUTS-3

individuals in our study population were still enrolled in education. By focusing on a younger population, future research could more closely assess the role of schools as a social space, and meeting place for groups with different educational backgrounds (with respect to family, neighbors and other networks).

\section{Conclusions}

We demonstrated the added value of a whole population network by studying patterns of segregation. Whole population networks cover the entire population and multiple layers of relationships which greatly improves the external 
validity of results. By including diverse types of relationships, whole population networks also provide greater insights than is possible with conventional methods that focus on residential or school segregation alone.

The results reaffirm many existing observations with regard to educational segregation but go beyond existing analysis in identifying the relative importance of specific layers. When weighted equally, the household layer on average increases the degree of segregation by education, which can be explained by homogamy. By contrast, work, neighbors and schools can provide greater exposure to individuals of differing educational backgrounds but this is context dependent as in some contexts work places and schools act as insulating institutions, increasing segregation. This approach to studying segregation at a population level using multiple layers allows these context dependencies to be better understood and interrogated, as the influence of specific types of institutions can be examined in different contexts.

Whole population networks more generally have diverse applications in social science and we would like to end by briefly summarizing these. This example analysis could be further extended by looking at how the exposure to various groups changes over time, whether polarization in society is evident or, in contrast, whether particular groups are becoming more integrated within wider society. Generating the network as a time-series is one of the next phases of development. All network layers include time markers that would allow for such networks to be expressed in continuous rather than discrete time.

Here we also focused on the exposure of individuals with differing educational qualifications but this approach could equally be used to examine the exposure to people from different ethnic groups or income levels. Segrega- 
tion and inequalities are a major concern of both social scientists and policy makers and their role in shaping broader social outcomes such as health, wellbeing and income is a large field of research. This approach, combining high

quality administrative data with advanced computational techniques could advance this field considerably.

The distribution of static population characteristics across a population is only one application of this approach. In the study of human behaviors it would be exceptionally valuable to identify the spread of behaviors or characteristics of individuals across a population. Network structure and dynamics can also be analyzed in much greater detail than current network analysis as the whole population network can be made dynamic over time. The potential applications are countless and could fundamentally shift the empirical base of many areas of social science.

This study was limited to the analysis of educational segregation in the Netherlands but there is no reason that it could not be extended to cover other topics or even other countries. The data requirements from the administrative data are considerable but not prohibitively so. So long as persistent identifiers are used across administrative data and adequate data protection is applied, this approach could be replicated in many countries.

\section{Acknowledgments}

This research was conducted using ODISSEI, the Open Data Infrastructure for Social Science and Economic Innovations (https://ror.org/03m8v6t10). Data and materials are available for replication via CBS Microdata Services and ODISSEI. Contact info@odissei-data.nl for more information. 


\section{References}

[1] Stephen P Borgatti, Ajay Mehra, Daniel J Brass, and Giuseppe Labianca. Network analysis in the social sciences. science, 323(5916):892$895,2009$.

[2] Marco van der Leij and Sebastian Buhai. A social network analysis of occupational segregation. 2008.

[3] Ashton M Verdery, Barbara Entwisle, Katherine Faust, and Ronald R Rindfuss. Social and spatial networks: Kinship distance and dwelling unit proximity in rural thailand. Social networks, 34(1):112-127, 2012.

[4] David MJ Lazer, Alex Pentland, Duncan J Watts, Sinan Aral, Susan Athey, Noshir Contractor, Deen Freelon, Sandra Gonzalez-Bailon, Gary King, Helen Margetts, et al. Computational social science: Obstacles and opportunities. Science, 369(6507):1060-1062, 2020.

[5] Anastasia Buyalskaya, Marcos Gallo, and Colin F Camerer. The golden age of social science. Proceedings of the National Academy of Sciences, 118(5), 2021.

[6] Bart F.M. Bakker, Johan Van Rooijen, and Leo Van Toor. The system of social statistical datasets of statistics netherlands: An integral approach to the production of register-based social statistics. Statistical Journal of the IAOS, 30(4):411-424, 2014.

[7] Pierre Bourdieu and Jean-Claude Passeron. Reproduction in education, society and culture, volume 4. Sage, 1990. 
[8] Diane Reay, Miriam E David, and Stephen J Ball. Degrees of choice: Class, race, gender and higher education. Trentham Books, 2005.

[9] Hans-Peter Blossfeld and Yossi Shavit. Persisting barriers. The Structure of Schooling: Readings in the Sociology of Education, 214, 2010.

[10] Shanto Iyengar, Yphtach Lelkes, Matthew Levendusky, Neil Malhotra, and Sean J Westwood. The origins and consequences of affective polarization in the united states. Annual Review of Political Science, 22:129$146,2019$.

[11] Willem R Boterman and Sako Musterd. Cocooning urban life: Exposure to diversity in neighbourhoods, workplaces and transport. Cities, 59:139-147, 2016.

[12] Duncan J Watts. Small worlds: the dynamics of networks between order and randomness. Princeton university press, 2004.

[13] Mark S Granovetter. The strength of weak ties. American journal of sociology, 78(6):1360-1380, 1973.

[14] Andrea Kavanaugh, Debbie Denise Reese, John M Carroll, and Mary Beth Rosson. Weak ties in networked communities. In Communities and technologies, pages 265-286. Springer, 2003.

[15] Sebastián Valenzuela, Teresa Correa, and Homero Gil de Zuniga. Ties, likes, and tweets: Using strong and weak ties to explain differences in protest participation across facebook and twitter use. Political communication, 35(1):117-134, 2018. 
[16] Gergő Tóth, Johannes Wachs, Riccardo Di Clemente, Ákos Jakobi, Bence Ságvári, János Kertész, and Balázs Lengyel. Inequality is rising where social network segregation interacts with urban topology. Nature communications, 12(1):1-9, 2021.

[17] Maarten Van Ham and William AV Clark. Neighbourhood mobility in context: household moves and changing neighbourhoods in the netherlands. Environment and planning A, 41(6):1442-1459, 2009.

[18] Thurston Domina. Brain drain and brain gain: Rising educational segregation in the united states, 1940-2000. City $\&$ Community, 5(4):387407, 2006.

[19] Tony Fielding. Migration in Britain: Paradoxes of the present, prospects for the future. Edward Elgar Publishing, 2012.

[20] Niels Kooiman, Jan Latten, and Marco Bontje. Human capital migration: A longitudinal perspective. Tijdschrift voor economische en sociale geografie, 109(5):644-660, 2018.

[21] Robert J Sampson. Great American city: Chicago and the enduring neighborhood effect. University of Chicago Press, 2012.

[22] Magnus Strömgren, Tiit Tammaru, Alexander M Danzer, Maarten van Ham, Szymon Marcińczak, Olof Stjernström, and Urban Lindgren. Factors shaping workplace segregation between natives and immigrants. Demography, 51(2):645-671, 2014.

[23] Christine R Schwartz. Pathways to educational homogamy in marital and cohabiting unions. Demography, 47(3):735-753, 2010. 
[24] Ted Mouw and Barbara Entwisle. Residential segregation and interracial friendship in schools. American Journal of sociology, 112(2):394-441, 2006.

[25] Federico Echenique and Roland G Fryer Jr. A measure of segregation based on social interactions. The Quarterly Journal of Economics, 122(2):441-485, 2007.

[26] Yang Xu, Alexander Belyi, Paolo Santi, and Carlo Ratti. Quantifying segregation in an integrated urban physical-social space. Journal of the Royal Society Interface, 16(160):20190536, 2019.

[27] Sergey V Buldyrev, Roni Parshani, Gerald Paul, H Eugene Stanley, and Shlomo Havlin. Catastrophic cascade of failures in interdependent networks. Nature, 464(7291):1025-1028, 2010.

[28] Gerardo Iñiguez, Federico Battiston, and Márton Karsai. Bridging the gap between graphs and networks. Communications Physics, 3(1):1-5, 2020 .

[29] UNESCO and UNESCO Institute for Statistics. International standard classification of education - ISCED 2011, 2012. http://uis.unesco.org/sites/default/files/documents/ international-standard-classification-of-education-isced-2011-en . pdf.

[30] NUTS - nomenclature of territorial units for statistics. https://ec. europa.eu/eurostat/web/nuts/background. Accessed: 2021-04-12. 
[31] Douglas S. Massey and Nancy A. Denton. The dimensions of residential segregation. Social Forces, 67(2):281-315, 1988.

[32] Coralio Ballester and Marc Vorsatz. Random walk-based segregation measures. Review of Economics and Statistics, 96:383-401, 2014.

[33] Leto Peel, Jean-Charles Delvenne, and Renaud Lambiotte. Multiscale mixing patterns in networks. Proceedings of the National Academy of Sciences, 115(16):4057-4062, Apr 2018.

[34] D. Jan van der Laan and Edwin de Jonge. Measuring local assortativity in the presence of missing values. In Complex Networks and their Applications VIII, pages 280-290. Springer, 2020.

[35] D. Jan van der Laan and Edwin de Jonge. Measuring segregation using a network of the dutch population. Paper presented at The 5th International Conference on Computational Social Science, 2019.

[36] Lloyd S. Shapley. A value for n-person games. Contributions to the Theory of Games, 2.28:307-317, 1953.

[37] Scott M Lundberg and Su-In Lee. A unified approach to interpreting model predictions. In I. Guyon, U. V. Luxburg, S. Bengio, H. Wallach, R. Fergus, S. Vishwanathan, and R. Garnett, editors, Advances in Neural Information Processing Systems 30, pages 4765-4774. Curran Associates, Inc., 2017.

[38] Douglas Bates and Martin Maechler. Matrix: Sparse and Dense Matrix Classes and Methods, 2021. R package version 1.3-2. 
[39] R Core Team. R: A Language and Environment for Statistical Computing. R Foundation for Statistical Computing, Vienna, Austria, 2021.

[40] Michel Scheerman, Narges Zarrabi, Martijn Kruiten, Maxime Mogé, Lykle Voort, Annette Langedijk, Ruurd Schoonhoven, and Tom Emery. Secure platform for processing sensitive data on shared hpc systems, 2021.

[41] Edwin Buitelaar, Anet BR Weterings, Otto Raspe, Olaf Jonkeren, and Willem Rogier Boterman. De verdeelde triomf: verkenning van stedelijkeconomische ongelijkheid en opties voor beleid: Ruimtelijke Verkenningen 2016. Planbureau voor de Leefomgevng, 2016.

[42] Sako Musterd. Social and ethnic segregation in europe: Levels, causes, and effects. Journal of urban affairs, 27(3):331-348, 2005.

[43] Hans-Peter Blossfeld. Educational assortative marriage in comparative perspective. Annual review of sociology, 35:513-530, 2009.

[44] Matthijs Kalmijn. Intermarriage and homogamy: Causes, patterns, trends. Annual review of sociology, 24(1):395-421, 1998. 